Research Article

\title{
Finite-Time Formation of Unmanned Aerial Vehicles with Switching Topologies and Disturbances: An Average Dwell Time Approach
}

\author{
Chao $M a \mathbb{D}^{1,2}$ and Wei Wu $\mathbb{D}^{2}$ \\ ${ }^{1}$ School of Automation and Electrical Engineering, University of Science and Technology Beijing, Beijing 100083, China \\ ${ }^{2}$ State Key Laboratory of Management and Control for Complex Systems, Institute of Automation, Chinese Academy of Sciences, \\ Beijing 100190, China \\ Correspondence should be addressed to Wei Wu; wei.wu@ia.ac.cn
}

Received 22 October 2018; Revised 14 January 2019; Accepted 11 February 2019; Published 28 April 2019

Academic Editor: Yue Wang

Copyright (C) 2019 Chao Ma and Wei Wu. This is an open access article distributed under the Creative Commons Attribution License, which permits unrestricted use, distribution, and reproduction in any medium, provided the original work is properly cited.

\begin{abstract}
This paper investigates the finite-time formation problem of unmanned aerial vehicles (UAVs) with switching topologies and external disturbances. The formation problem is first transformed into the finite-time stability and bounded problems of a switched system, respectively. In particular, the finite-time unachievable topology is concerned for unreliable information exchanges. By employing the average dwell time (ADT) method, sufficient criteria are established to deal with the switching topologies such that the desired time-varying formation can be achieved in finite time. Then, the topology-dependent controller can be designed in terms of matrix technique. Finally, an illustrative example is given to verify the effectiveness of our proposed formation strategy.
\end{abstract}

\section{Introduction}

During the past decade, the unmanned aerial vehicles (UAVs) have grown from academic researches into practical applications capable of various missions in both civilian and military areas [1-3]. Recently, with the substantial progresses in swarm intelligence, cooperation issues of multiple UAVs and robots have been receiving increasing attention for distinguishing advantages. Based on information exchanges according to the communication topology, a group of UAVs can work collectively to meet the need for high robustness, reliability, flexibility, and efficiency [4-10]. In particular, the formation problem is one of the most hot research topics for UAVs, which means that the UAVs can keep a certain flying configuration while accomplishing the tasks. Note that the communication among the UAVs can play a very significant role in formation since the information exchanges are conducted via the communication networks according to the communication topology [11-15]. However, it is worth mentioning that the communication topology may change at time due to unreliable communication networks or security reason, and this may lead to unpredictable mission failure or performance degradation. As a consequence, the UAVs with switching topologies are of theoretical importance and practical background. So far, there are different remarkable approaches reported in the literature and the references therein [16-18].

On another research front line, the switched systems have been receiving increasing attention due to their novel ability in describing practical systems with mode switchings [1922]. In particular, as one of the most effective analysis and synthesis methods for switched systems, the average dwell time (ADT) has been widely utilized. In comparison to the dwell time method, the ADT can further reduce the conservatism for each subsystem [23-25]. Therefore, a natural question arises from the prior discussions: can we introduce 
the merit of ADT of switched systems to deal with the switching topologies? The answer to this question is challenging, but the efforts can lead to encouraging results. Furthermore, in most applications, the stability is required to be achieved over a finite-time interval, which gives rises to the researches on finite-time stability, and burgeoning investigations on finite-time consensus problems of multiagent systems with switching topologies have been made [26-28]. However, it is worth mentioning that the unachievable switching topologies are not concerned in most existing results. It is noted that there is also a need for the finite-time formation of UAVs by taking into account its realistic background. Until now, to the best of the authors' knowledge, there still exists a considerable gap in solving the formation problem of UAVs with switching topologies based on the switched system theory, let alone those with unachievable topologies. The aim of this article is to make one of the first attempts to shorten such a gap.

Inspired by the ADT method in switched systems, in this paper, the finite-time formation of UAVs with switching topologies and disturbances is studied in terms of the ADT approach. Compared with the existing results, the main contributions of our paper can be summarized as follows. (1) The finite-time formation problem of UAVs with unachievable switching topologies is investigated for the first time. More precisely, the switching topologies are categorized into two types: the achievable topologies and the unachievable topologies. The standard of category relies on whether the finitetime formation can be reached for a fixed topology. For example, it can be verified that the directed topology without a directed spanning tree is unachievable topology. This can further extend the applications of switching topologies and is more practical for UAVs. It can be found that the established results with unachievable topologies are more practical in the applications. (2) The disturbances of UAVs are also considered for formation robustness with the finitetime boundedness problem. On the basis of ADT with the multiple Lyapunov function (MLF) method, sufficient conditions are established for ensuring the finite-time formation and the desired topology-dependent controller gains are obtained accordingly.

The remainder of our paper is given as follows: Section 2 introduces the finite-time formation control problem of UAVs with some essential preliminaries. In Section 3, the main analysis and synthesis procedures are provided with the ADT method. Section 4 presents the numerical example to demonstrate the effectiveness of our proposed formation strategies. Finally, the paper is concluded in Section 5.

Notation 1. $\mathbb{R}^{n}$ and $\mathbb{R}^{m} \times n$ denote the $n$ dimensional Euclidean space and the space of $m \times n$ real matrices, respectively. $A-B>0(A-B \prec 0)$ denotes that $A-B$ is positive definite (negative definite). $\lambda_{\max }(A)$ and $\lambda_{\min }(A)$ denote the maximum and minimum eigenvalues of matrix $A$, respectively. $\mathscr{L}_{2}[0, \infty)$ denotes the space of squareintegrable vector functions over $[0, \infty) . A \otimes B$ stands for the Kronecker product. $*$ represents the ellipsis symmetry terms in symmetric block matrices. All matrices are compatible for algebraic operations.

\section{Problem Formulation and Preliminaries}

2.1. The UAV Dynamics. Consider a group of UAVs with the following kinematics and dynamics:

$$
\begin{aligned}
& \left\{\begin{array}{l}
\dot{x}_{i}=v_{i} \cos a_{i} \cos b_{i}, \\
\dot{y}_{i}=v_{i} \cos a_{i} \sin b_{i}, \\
\dot{z}_{i}=v_{i} \sin a_{i},
\end{array}\right. \\
& \left\{\begin{array}{l}
\dot{v}_{i}=\frac{T_{i}-D_{i}}{m_{i}}-g \sin a_{i}-d_{1 i}, \\
\dot{a}_{i}=\frac{g}{v_{i}}\left(n_{g i} \cos \phi_{i}-\cos a_{i}\right)+d_{2 i}, \\
\dot{b}_{i}=\frac{L_{i} \sin \phi_{i}}{m_{i} v_{i} \cos a_{i}}+d_{3 i},
\end{array}\right.
\end{aligned}
$$

where $\left[x_{i}, y_{i}, z_{i}\right]^{T}$ denotes the position of the $i$ th UAV, $v_{i}$ represents the ground speed, $a_{i}$ stands for the flight-path angle, $b_{i}$ is the heading angle, $T_{i}$ denotes the engine thrust, $D_{i}$ represents the drag, $m_{i}$ denotes the mass, $g$ stands for the acceleration due to gravity, $L_{i}$ is the lift force, $n_{g i}=$ $L_{i} / m_{i} g$ denotes the $g$-load value, $\phi_{i}$ represents the banking angle, and $d_{k i}, k=1,2,3$ stands for the vortex disturbances.

By applying the feedback linearization method as

$$
\left\{\begin{array}{l}
\ddot{x}_{i}=u_{x i}+w_{x i}, \\
\ddot{y}_{i}=u_{y i}+w_{y i}, \\
\ddot{z}_{i}=u_{z i}+w_{z i},
\end{array}\right.
$$

where $\left[u_{x i}, u_{y i}, u_{z i}\right]^{T}$ denotes the virtual acceleration control input and $\left[w_{x i}, w_{y i}, w_{z i}\right]^{T}$ represents the disturbances, the relationships between virtual control variables and the actual control variables are given by

$$
\left\{\begin{array}{l}
T_{i}=m i\left[\left(u_{z i}+g\right) \sin a_{i}+u_{x y i} \cos a_{i}\right]+D_{i}, \\
n_{g i}=\frac{\left(u_{z i}+g\right) \cos a_{i}-u_{x y i} \sin a_{i}}{g \cos \phi_{i}}, \\
\phi_{i}=\arctan \left[\frac{u_{y i} \cos b_{i}-u_{x i} \sin b_{i}}{\left(u_{z i}+g\right) \cos a_{i}-u_{x y i} \sin a_{i}}\right],
\end{array}\right.
$$

with $u_{x y i}=u_{x i} \cos b_{i}+u_{y i} \sin b_{i}, a_{i}=\arcsin \left(\dot{z}_{i} / v_{i}\right)$, and $b_{i}=$ $\arctan \left(\dot{y}_{i} / \dot{x}_{i}\right)$.

Consequently, by denoting

$$
\left\{\begin{array}{l}
\xi_{i}(t)=\left[x_{i}, y_{i}, z_{i}, \dot{x}_{i}, \dot{y}_{i}, \dot{z}_{i}\right]^{T}, \\
u_{i}(t)=\left[u_{x i}, u_{y i}, u_{z i}\right]^{T}, \\
w_{i}(t)=\left[w_{x i}, w_{y i}, w_{z i}\right]^{T},
\end{array}\right.
$$


the UAV dynamics can be obtained as follows:

$$
\dot{\xi}_{i}(t)=A \xi_{i}(t)+B u_{i}(t)+B w_{i}(t)
$$

where

$$
\begin{aligned}
& A=\left[\begin{array}{ll}
0 & I_{3} \\
0 & 0
\end{array}\right], \\
& B=\left[\begin{array}{l}
0 \\
I_{3}
\end{array}\right] .
\end{aligned}
$$

2.2. Graph Theory. The directed graph $\mathscr{G}=\{\mathscr{V}, \mathscr{E}, \mathscr{A}\}$ is adopted to describe the communication topology of the UAVs. $\mathscr{A}=\left[a_{i j}\right] \in \mathbb{R}^{N \times N}$ represents the weighted adjacency matrix with

$$
\left\{\begin{array}{l}
a_{i j}>0\left(v_{i}, v_{j}\right) \in \mathscr{E}, \\
a_{i j}=0, \quad \text { otherwise. }
\end{array}\right.
$$

$\mathscr{V}(\mathscr{G})=\left\{v_{1}, \cdots, v_{N}\right\}$ and $\mathscr{E}$ denote the sets of nodes and edges, respectively. The $L=\left(l_{i j}\right)_{N \times N}$ denotes the Laplacian matrix with

$$
\left\{\begin{array}{l}
l_{i j}=-a_{i j}, \quad i \neq j, \\
l_{i i}=\sum_{j=1, i \neq j}^{N} a_{i j} .
\end{array}\right.
$$

If $\mathscr{G}$ has a directed spanning tree, then 0 is the eigenvalue of $L$. More details of the algebraic graph theory can be found in [29].

The switching topologies are studied in this paper. Let $\left\{\mathscr{G}_{\sigma(t)}: \sigma(t) \in \mathcal{S}=1,2, \ldots, n\right\}$ denote all possible switching graphs, where $\sigma(t)$ represents the index set for all switching graphs. The switching signal $\sigma(t):[0, \infty) \rightarrow p$, and its value is the index of the graph at time $t$. The switching sequence is $t_{1}, t_{2}, \ldots$, where $\left[t_{h}, t_{h+1}\right), h=1,2, \ldots$, is uniformly bounded nonoverlapping [30].

Moreover, for any possible $L_{\sigma(t)} \in \mathbb{R}^{N \times N}$ of graph $\mathscr{G}_{\sigma(t)}$ and a full row rank matrix $E \in \mathbb{R}^{(N-1) \times N}$ defined as

$$
E=\left[\begin{array}{ccccc}
1 & -1 & 0 & \cdots & 0 \\
0 & 1 & -1 & \cdots & 0 \\
\vdots & \vdots & \ddots & \vdots & \vdots \\
0 & 0 & 0 & 1 & -1
\end{array}\right]
$$

there exists a matrix $M_{\sigma(t)} \in \mathbb{R}^{N \times(N-1)}$ such that $L_{\sigma(t)}=$ $M_{\sigma(t)} E$.

Remark 1. It is worth mentioning that when $\mathscr{G}_{\sigma(t)}$ is achievable $\left(L_{\sigma(t)}\right.$ has a directed spanning tree), $M_{\sigma(t)}$ is of full column rank and the eigenvalues of $E M_{\sigma(t)}$ are the same as that of $L_{\sigma(t)}$. When $\mathscr{G}_{\sigma(t)}$ is unachievable, the above conditions of $M_{\sigma(t)}$ will not be satisfied.

Without loss of generality, suppose that there are $r$ achievable topologies with $\sigma(t)=1,2, \ldots, r$ which means that the formation can be achieved with the $L_{\sigma(t)}$ and $n-r$ unachievable topologies with $r+1$ and $r+2, \ldots, n$, otherwise. All the possible communication topologies can be divided into the achievable set denoted as $\mathscr{A} \mathscr{T}$ and the unachievable set denoted as $\mathscr{U} \mathscr{A} \mathscr{T}$.

2.3. Finite-Time Formation. For the time-varying formation problem, denote $h(t)=\left[h_{1}(t), h_{2}(t), \ldots, h_{N}(t)\right]^{T} \in \mathbb{R}^{6 N}$, where $h_{i}(t)=\left[h_{x i}(t), h_{y i}(t), h_{z i}(t), h_{v x i}(t), h_{v y i}(t), h_{v y i}(t), h_{v z i}\right.$ $(t)]^{T}$ is a piecewise continuously differentiable, and $e(t)=$ $\left[e_{1}(t), e_{2}(t), \ldots, e_{N}(t)\right]^{T} \in \mathbb{R}^{6 N}$, where $e_{i}(t)=\xi_{i}(t)-h_{i}(t)$.

Consequently, the formation controller for each UAV can be designed as follows:

$$
\begin{aligned}
u_{i}(t)= & K_{1 \sigma(t)}\left(\xi_{i}(t)-h_{i}(t)\right)+K_{2 \sigma(t)} \sum_{j=1}^{N} a_{i j \sigma(t)}\left(\left(\xi_{i}(t)-h_{i}(t)\right)\right. \\
& \left.-\left(\xi_{i}(t)-h_{j}(t)\right)\right)+\mu_{i h}(t), \quad i=1,2, \ldots, N,
\end{aligned}
$$

where $\dot{h}_{i}(t)=A h_{i}(t)+B \mu_{i h}(t)$ and $K_{1 \sigma(t)}$ and $K_{2 \sigma(t)}$ are topology-dependent controller gains to be determined, respectively.

As a result, the closed-loop dynamics of the UAVs can be obtained by

$$
\begin{aligned}
\dot{\xi}(t)= & (I \otimes A) \xi(t)+\left(I \otimes B K_{1 \sigma(t)}\right)(\xi(t)-h(t)) \\
& +\left(L_{\sigma(t)} \otimes B K_{2 \sigma(t)}\right) \times(\xi(t)-h(t)) \\
& +(I \otimes B) \mu_{h}(t)+(I \otimes B) w(t),
\end{aligned}
$$

where $\mu_{h}(t)=\left[\mu_{1 h}^{T}(t), \mu_{2 h}^{T}(t), \ldots, \mu_{N h}^{T}(t)\right]^{T}$.

In addition, note that $e(t)=\xi(t)-h(t)$; then, it follows that

$$
\begin{aligned}
\dot{e}(t)= & (I \otimes A) e(t)+\left(I \otimes B K_{1 \sigma(t)}\right) e(t) \\
& +\left(L_{\sigma(t)} \otimes B K_{2 \sigma(t)}\right) e(t)+(I \otimes B) w(t) .
\end{aligned}
$$

Premultiplying both sides of (13) by $(E \otimes I)$ yields that

$$
\begin{aligned}
\dot{\varepsilon}(t)= & (I \otimes A) \varepsilon(t)+\left(I \otimes B K_{1 \sigma(t)}\right) \varepsilon(t) \\
& +\left(E M_{\sigma(t)} \otimes B K_{2 \sigma(t)}\right) \varepsilon(t)+(I \otimes B) \omega(t),
\end{aligned}
$$

where $\varepsilon(t)=(E \otimes I) e(t)$ denotes the disagreement of $e(t)$ and $\omega(t)=(E \otimes I) w(t)$, respectively.

To this end, the following definitions are given for later use. 
Definition 1 (average dwell time, ADT) [31]. For any $T>t$ $\geq 0$, denote $N_{\sigma}(T, t)$ as the number of switching over $[t, T]$. $\sigma(t)$ is said to have an average dwell time (ADT) $\tau_{a}>0$, if there exist positive numbers $N_{0}$ such that

$$
N_{\sigma}(T, t) \leq N_{0}+\frac{T-t}{\tau_{a}}
$$

Definition 2 (finite-time formation, FTF) [32]. Given constants $c_{1}>0, c_{2}>0$, and $T>0$, with $c_{1}<c_{2}$, matrix $R>0$, and a switching signal $\sigma(t) \in \mathcal{S}$, the finite-time formation is said to be achieved with $\left(c_{1}, c_{2}, T, R, \sigma(t)\right)$, if it holds that

$$
\varepsilon^{T}(0) R \varepsilon(0) \leq c_{1} \Rightarrow \varepsilon^{T}(t) R \varepsilon(t) \leq c_{2}, \quad \forall t \in(0, T] .
$$

Definition 3 (uniformly finite-time formation, UFTF) [32]. Given constants $c_{1}>0, c_{2}>0$, and $T>0$, with $c_{1}<c_{2}$ and matrix $R>0$, the uniformly finite-time formation is said to be achieved with $\left(c_{1}, c_{2}, T, R\right)$, if it holds that

$$
\varepsilon^{T}(0) R \varepsilon(0) \leq c_{1} \Rightarrow \varepsilon^{T}(t) R \varepsilon(t) \leq c_{2}, \quad \forall t \in(0, T] .
$$

Definition 4 (finite-time bounded formation, FTBF) [32]. Given constants $c_{1}>0, c_{2}>0, d_{w} \geq 0$, and $T>0$, with $c_{1}<$ $c_{2}$, matrix $R>0$, and a switching signal $\sigma(t) \in S$, the finitetime bounded formation is said to be achieved with $\left(c_{1}, c_{2}\right.$, $\left.T, R, \sigma(t), d_{w}\right)$, if it holds that

$$
\begin{gathered}
\varepsilon^{T}(0) R \varepsilon(0) \leq c_{1} \Rightarrow \varepsilon^{T}(t) R \varepsilon(t) \leq c_{2}, \quad \forall t \in(0, T] \\
\forall w(t): \int_{0}^{T} w^{T}(t) w(t) d t \leq d_{w} .
\end{gathered}
$$

Definition 5 (uniformly finite-time bounded formation, UFTBF) [32]. Given constants $c_{1}>0, c_{2}>0, d_{w} \geq 0$, and $T$ $>0$, with $c_{1}<c_{2}$ and matrix $R>0$, the uniformly finite-time bounded formation is said to be achieved with $\left(c_{1}, c_{2}, T, R\right.$, $d_{w}$ ), if for any switching signal $\sigma(t) \in \mathcal{S}$ it holds that

$$
\begin{gathered}
\varepsilon^{T}(0) R \varepsilon(0) \leq c_{1} \Rightarrow \varepsilon^{T}(t) R \varepsilon(t) \leq c_{2}, \quad \forall t \in(0, T], \\
\forall w(t): \int_{0}^{T} w^{T}(t) w(t) d t \leq d_{w} .
\end{gathered}
$$

Remark 2. It should be pointed out that the concept of finitetime stability is different from Lyapunov asymptotic stability. A system can be finite-time stable but not Lyapunov asymptotically stable and vice versa.

\section{Main Results}

In this section, the FTF and the FTBF problems of UAVs are solved with details.

\subsection{Finite-Time Formation without Disturbances}

Theorem 1. Consider the group of UAVs (1) with switching topologies, for giving constants $\alpha_{i} \geq 0, \beta_{i} \geq 0, i \in \mathcal{S}, \mu>1$, and the topology-dependent controller gains $K_{1 i}$ and $K_{2 i}$, if there exist matrices $P_{i}>0, \forall(i, j) \in \mathcal{S} \times \mathcal{S}, i \neq j$, such that

$$
\begin{gathered}
\prod_{i}-a_{i}\left(I \otimes P_{i}\right) \prec 0, \quad i \in \mathscr{A} \mathscr{T}, \\
\prod_{i} \beta_{i}\left(I \otimes P_{i}\right) \prec 0, \quad i \in \mathcal{U} \mathscr{A} \mathscr{T}, \\
P_{i} \preceq \mu P_{j}, \quad i \neq j,
\end{gathered}
$$

where

$$
\prod_{i}:=\operatorname{sym}\left\{\left(I \otimes P_{i} A\right)+\left(I \otimes P_{i} B K_{1 i}\right)+\left(E M_{i} \otimes P_{i} B K_{2 i}\right)\right\}
$$

and the total dwell time of the unachievable topologies $T^{+}$ satisfies

$$
T^{+}(0, t)<\frac{\ln c_{2} \lambda_{\min }\left(P_{i}\right) \lambda_{\min }(R)-\ln c_{1} \lambda_{\max }(R) \lambda_{\max }\left(P_{i}\right)-N \ln \mu-a T}{\beta-a} .
$$

Then, the FTF can be achieved with respect to $\left(c_{1}, c_{2}, \mathrm{~T}\right.$, $\mathrm{R}, \sigma(t))$
Furthermore, when $T^{+}$can be fixed, the switching signal has an ADT satisfying

$$
\tau_{a} \geq \tau_{a}^{*}=\frac{T \ln \mu}{\ln c_{2} \lambda_{\min }\left(P_{i}\right) \lambda_{\min }(R)-\ln c_{1} \lambda_{\max }(R) \lambda_{\max }\left(P_{i}\right)-a T-(\beta-a) T^{+}(0, t)} .
$$


Proof 1. Choose the multiple Lyapunov-like functions as follows:

$$
V_{\sigma(t)}(t)=\varepsilon^{T}(t)\left(I \otimes P_{\sigma(t)}\right) \varepsilon(t)
$$

Then, the LMIs (20) and (21) mean that

$$
\begin{array}{ll}
\dot{V}_{i}(t) \prec \alpha_{i} V_{i}(t), & i \in \mathscr{A} \mathscr{T}, \\
\dot{V}_{i}(t) \prec \beta_{i} V_{i}(t), & i \in \mathscr{U} \mathscr{A} \mathscr{T} .
\end{array}
$$

For $t \in\left[t_{k}, t_{k}+1\right)$, it can be obtained by (27) that

$$
\begin{array}{ll}
V(t)<e^{a_{\sigma\left(t_{k}\right)}\left(t-t_{k}\right)} V_{\sigma(t)}\left(t_{k}\right), & \sigma\left(t_{k}\right) \in \mathscr{A} \mathscr{T}, \\
V(t)<e^{\beta_{\sigma\left(t_{k}\right)}\left(t-t_{k}\right)} V_{\sigma(t)}\left(t_{k}\right), & \sigma\left(t_{k}\right) \in \mathcal{U} \mathscr{A} \mathscr{T} .
\end{array}
$$

Without loss of generality, denote $\sigma\left(t_{k}\right)=i$ and $\sigma\left(t_{k}^{-}\right)=j$ with $N_{0}=0$. Then, for any $i \neq j$, if (22) holds then

$$
V_{\sigma\left(t_{k}\right)}\left(t_{k}\right) \leq \mu V_{\sigma\left(t_{k}^{-}\right)}\left(t_{k}^{-}\right)
$$

Thus, for $t \in[0, T)$, letting $a=\max _{i \in \mathcal{S}}\left(a_{i}\right)$ and $\beta=$ $\max _{i \in \mathcal{S}}\left(\beta_{i}\right)$ yields that

$$
\begin{aligned}
V(t) & <e^{a T^{-}(0, t)+\beta T^{+}(0, t)} V_{\sigma(t 0)}(0) \\
& =\mu^{N} e^{a\left(T-T^{+}(0, t)\right)+\beta T^{+}(0, t)} V_{\sigma(0)}(0),
\end{aligned}
$$

where $N$ is the switching number of $\sigma(t)$ over $[0, T)$ and $T^{+}(0, t)$ is the total dwell time of $\mathscr{U} \mathscr{A} \mathscr{T}$.

On the other hand, it holds that

$$
\begin{aligned}
\lambda_{\min }\left(P_{\sigma(t)}\right) \varepsilon^{T}(t) \varepsilon(t) & \leq V_{\sigma(t)}(t) \\
= & \varepsilon^{T}(t) P_{\sigma(t)} \varepsilon(t) \leq \lambda_{\max }\left(P_{\sigma(t)}\right) \varepsilon^{T}(t) \varepsilon(t),
\end{aligned}
$$

which implies that when the FTF in Definition 2 can be achieved it can be verified that

$$
\begin{aligned}
\varepsilon^{T}(t) R \varepsilon(t) & \leq \lambda_{\max }(R) \varepsilon^{T}(t) \varepsilon(t) \\
& \leq \lambda_{\max }(R) \frac{V_{\sigma(t)}(t)}{\lambda_{\min }\left(P_{\sigma(t)}\right)} \\
& <\frac{\lambda_{\max }(R)}{\lambda_{\min }\left(P_{\sigma(t)}\right)} \mu^{N} e^{a\left(T-T^{+}(0, t)\right)+\beta T^{+}(0, t)} V_{\sigma\left(t_{0}\right)}(0) \\
& \leq \frac{\lambda_{\max }(R) \lambda_{\max }\left(P_{\sigma(t)}\right)}{\lambda_{\min }\left(P_{\sigma(t)}\right)} \mu^{N} e^{a T} e^{\left.(\beta-a) T^{+}(0, t)\right)} \varepsilon^{T}(0) \varepsilon(0) \\
& \leq c_{1} \mu^{N} \frac{\lambda_{\max }(R) \lambda_{\max }\left(P_{\sigma(t)}\right)}{\lambda_{\min }\left(P_{\sigma(t)}\right) \lambda_{\min }(R)} e^{a T} e^{\left.(\beta-a) T^{+}(0, t)\right)}<c_{2} .
\end{aligned}
$$

Moreover, the ADT $\tau_{a}$ can be obtained for a fixed $T^{+}(0, t)$ according to Definition 1, which completes the proof.

Based on the established conditions in Theorem 1, Theorem 2 is given to calculate the topology-dependent controller gains.

Theorem 2. Consider the group of UAVs (1) with switching topologies, for giving constants $\alpha_{i} \geq 0, \beta_{i} \geq 0, i \in \mathcal{S}$, and $\mu>$ 1 , if there exist matrices $P_{i}>0$ and $W_{2 i}, \forall(i, j) \in \mathcal{S} \times \mathcal{S}, i \neq j$, such that

$$
\begin{aligned}
\Xi_{i}-\alpha_{i}\left(I \otimes \tilde{P}_{i}\right)<0, \quad i \in \mathscr{A} \mathscr{T}, \\
\Xi_{i}-\beta_{i}\left(I \otimes \tilde{P}_{i}\right)<0, \quad i \in \mathscr{U} \mathscr{A} \mathscr{T}, \\
\tilde{P}_{i} \preceq \mu \tilde{P}_{j}, \quad i \neq j,
\end{aligned}
$$

where

$$
\Xi_{i}:=\operatorname{sym}\left\{\left(I \otimes A \tilde{P}_{i}\right)+\left(I \otimes B K_{1 i} \tilde{P}_{i}\right)+\left(E M_{i} \otimes B W_{2 i}\right)\right\},
$$

and the total dwell time of the unachievable topologies $T^{+}$ satisfies

$$
T^{+}(0, t)<\frac{\ln c_{2} \lambda_{\min }\left(\tilde{P}_{i}^{-1}\right) \lambda_{\min }(R)-\ln c_{1} \lambda_{\max }(R) \lambda_{\max }\left(\tilde{P}_{i}^{-1}\right)-N \ln \mu-a T}{\beta-a} .
$$

Then, the FTF can be achieved with respect to $\left(c_{1}, c_{2}, T\right.$, $R, \sigma(t))$.
Furthermore, when $T^{+}$can be fixed, the switching signal has an ADT satisfying

$$
\tau_{a} \geq \tau_{a}^{*}=\frac{T \ln \mu}{\ln c_{2} \lambda_{\min }\left(\tilde{P}_{i}^{-1}\right) \lambda_{\min }(R)-\ln c_{1} \lambda_{\max }(R) \lambda_{\max }\left(\tilde{P}_{i}^{-1}\right)-a T-(\beta-a) T^{+}(0, t)} .
$$


In addition, when the topology-dependent controller gain $K_{1 i}$ is configured, $K_{2 i}$ can be obtained by

$$
K_{2 i}=W_{2 i} \tilde{P}_{i}^{-1}
$$

Proof 2. By letting $\tilde{P}_{i}=P_{i}^{-1}$ and $W_{2 i}=K_{2 i} \tilde{P}_{i}$ and performing matrix congruent transformation, the conditions then follow directly from Theorem 1 .

Remark 3. It is noted that $K_{1 i}$ is related with the motion modes of the formation center, such that $K_{1 i}$ is generally separately designed in advance of $K_{2 i}$. When $K_{1 i}$ is configured appropriately, then the desired $K_{2 i}$ can be obtained by solving the conditions in Theorem 2 accordingly. For the large-scale UAVs, since the formation configuration $h(t)$ is prescribed, the controllers of UAVs can be designed according to $u_{i}(t)$, where the information exchanges among the UAVs are also considered.

Note that if a common Lyapunov-like function can be found for (6), Corollary 1 can be derived.

Corollary 1. Consider the group of UAVs (1) with switching topologies, for giving constants $\alpha_{i} \geq 0$ and $\beta_{i} \geq 0, i \in \mathcal{S}$, if there exist matrices $P_{i}>0$ and $W_{2 i}, \forall(i, j) \in \mathcal{S} \times \mathcal{S}, i \neq j$, such that

$$
\begin{aligned}
& \Xi_{i}-\alpha\left(I \otimes \tilde{P}_{i}\right)<0, \quad i \in \mathscr{A} \mathscr{T}, \\
& \Xi_{i}-\beta\left(I \otimes \tilde{P}_{i}\right), \quad i \in \mathscr{U} \mathscr{A} \mathscr{T},
\end{aligned}
$$

and the total dwell time of the unachievable topologies $T^{+}$ satisfies

$$
T^{+}(0, t)<\frac{\ln c_{2} \lambda_{\min }\left(\tilde{P}_{i}^{-1}\right) \lambda_{\min }(R)-\ln c_{1} \lambda_{\max }(R) \lambda_{\max }\left(\tilde{P}_{i}^{-1}\right)-a T}{\beta-a} .
$$
$T, R)$.

Then, the UFTF can be achieved with respect to $\left(c_{1}, c_{2}\right.$,

In addition, when the topology-dependent controller gain $K_{1 i}$ is configured, $K_{2 i}$ can be obtained by

$$
K_{2 i}=W_{2 i} \tilde{P}_{i}^{-1}
$$

3.2. Finite-Time Formation with Disturbances. When the external disturbances of UAVs are considered, the theorems of FTBF and UFTBF are presented.

Theorem 3. Consider the group of UAVs (1) with switching topologies and disturbances, for giving constants $\alpha_{i} \geq 0, \beta_{i} \geq$ $0, i \in \mathcal{S}, \mu>1, \kappa_{1 i}>0, \kappa_{2 i}>0$, and the topology-dependent controller gains $K_{1 i}$ and $K_{2 i}$, if there exist matrices $P_{i}>0$, $\forall(i, j) \in \mathcal{S} \times \mathcal{S}, i \neq j$, such that

$$
\left[\begin{array}{cc}
\Psi_{1 i} & \left(I \otimes P_{i} B\right) \\
* & -\kappa_{1 i} I
\end{array}\right]<0, \quad i \in \mathscr{A} \mathscr{T}
$$

$$
\begin{gathered}
{\left[\begin{array}{cc}
\Psi_{2 i} & \left(I \otimes P_{i} B\right) \\
* & -\kappa_{2 i} I
\end{array}\right]<0, \quad i \in \mathscr{U} \mathscr{A} \mathscr{T},} \\
P_{i} \preceq \mu_{i} P_{j},
\end{gathered}
$$

where

$$
\begin{aligned}
\Psi_{1 i}:= & \operatorname{sym}\left\{\left(I \otimes P_{i} A\right)+\left(I \otimes P_{i} B K_{1 i}\right)+\left(E M_{i} \otimes P_{i} B K_{2 i}\right)\right\} \\
& -\alpha_{i}\left(I \otimes P_{i}\right), \\
\Psi_{2 i}:= & \operatorname{sym}\left\{\left(I \otimes P_{i} A\right)+\left(I \otimes P_{i} B K_{1 i}\right)+\left(E M_{i} \otimes P_{i} B K_{2 i}\right)\right\} \\
& -\beta_{i}\left(I \otimes P_{i}\right),
\end{aligned}
$$

and the total dwell time of the unachievable topologies $T^{+}$ satisfies

$$
T^{+}(0, t)<\frac{\ln \left(c_{2} \lambda_{\min }\left(P_{i}\right) \lambda_{\min }(R)-\mu^{N} \lambda_{\max }(R) \lambda_{\max }\left(P_{i}\right) \lambda_{\min }(R) \kappa e^{\beta T} d_{w}\right)}{\beta-a}-\frac{N \ln \mu-\ln c_{1} \lambda_{\max }(R) \lambda_{\max }\left(P_{i}\right)-a T}{\beta-a}
$$

Then, the FTBF can be achieved with respect to $\left(c_{1}, c_{2}, T\right.$, $\left.R, \sigma(t), d_{w}\right)$.
Furthermore, when $T^{+}$can be fixed, the switching signal has an $A D T$ satisfying 


$$
\tau_{a}>\tau_{a}^{*}=\frac{T \ln \mu}{\ln c_{2} \lambda_{\min }\left(P_{i}\right) \lambda_{\min }(R)-\ln \left(\lambda_{\max }(R) \lambda_{\max }\left(P_{i}\right)\left(e^{a\left(T-T^{+}(0, t)\right)+\beta T^{+}(0, t)} c_{1}+\lambda_{\min }(R) \kappa e^{\beta T} d_{w}\right)\right)} .
$$

Proof 3. Select the multiple Lyapunov-like function as follows:

$$
V_{i}(t)=\varepsilon^{T}(t)\left(I \otimes P_{i}\right) \varepsilon(t)
$$

Then, the LMIs (41) and (42) mean that

$$
\begin{array}{ll}
\dot{V}_{i}(t) \prec \alpha_{i} V_{i}(t)+\kappa_{1 i} \omega^{T}(t) \omega(t), & i \in \mathscr{A} \mathscr{T}, \\
\dot{V}_{i}(t) \prec \beta_{i} V_{i}(t)+\kappa_{2 i} \omega^{T}(t) \omega(t), & i \in \mathscr{U} \mathscr{A} \mathscr{T} .
\end{array}
$$

Following same lines in the proof of Theorem 1, for $t \in\left[t_{k}, t_{k+1}\right)$, it holds that

$$
\begin{aligned}
V(t)< & e^{a_{\sigma\left(t_{k}\right)}\left(t-t_{k}\right)} \mu V_{\sigma\left(t_{k}^{-}\right)}\left(t_{k}^{-}\right) \\
& +\int_{t_{k}}^{t} e^{a_{\sigma\left(t_{k}\right)}(t-s)} \kappa_{1 \sigma\left(t_{k}\right)} \omega^{T}(s) \omega(s) d s, \quad \sigma\left(t_{k}\right) \in \mathscr{A} \mathscr{T}, \\
V(t)<e^{\beta_{\sigma(t)}\left(t-t_{k}\right)} \mu V_{\sigma\left(t_{k}^{-}\right)}\left(t_{k}^{-}\right) & \\
& +\int_{t_{k}}^{t} e^{\beta_{\sigma\left(t_{k}\right)}(t-s)} \kappa_{2 \sigma\left(t_{k}\right)} \omega^{T}(s) \omega(s) d s, \quad \sigma\left(t_{k}\right) \in \mathcal{U A T} .
\end{aligned}
$$

By letting $\pi_{\sigma(t)}=a_{\sigma(t)}, \kappa_{\sigma(t)}=\kappa_{1 \sigma(t)}$, and $\sigma(t) \in \mathscr{A} \mathscr{T}$ and $\pi_{\sigma(t)}=\beta_{\sigma(t)}, \kappa_{\sigma(t)}=\kappa_{2 \sigma(t)}$, and $\sigma(t) \in \mathscr{U} \mathscr{A} \mathscr{T}$, one has

$$
\begin{aligned}
V(t) & \leq e^{\pi_{\sigma\left(t_{k}\right)}\left(t-t_{k}\right)} \mu V_{\sigma\left(t_{k}^{-}\right)}\left(t_{k}^{-}\right)+\int_{t_{k}}^{t} e^{\pi_{\sigma\left(t_{k}\right)}(t-s)} \kappa_{\sigma\left(t_{k}\right)} \omega^{T}(s) \omega(s) d s \\
& \leq e^{\pi_{\sigma\left(t_{k}\right)}\left(t-t_{k}\right)} \mu\left(e^{\pi_{\sigma\left(t_{k-1}\right)}\left(t_{k}-t_{k-1}\right)} \mu V_{\sigma\left(t_{k-1}^{-}\right)}\left(t_{k-1}^{-}\right)\right.
\end{aligned}
$$

$$
\begin{aligned}
& \left.\quad+\int_{t_{k-1}}^{t} e^{\pi_{\sigma\left(t_{k-1}\right)}\left(t_{k}-s\right)} \kappa_{\sigma\left(t_{k-1}\right)} \omega^{T}(s) \omega(s) d s\right) \\
& +\int_{t_{k}}^{t} e^{\pi_{\sigma\left(t_{k}\right)}(t-s)} \kappa_{\sigma\left(t_{k}\right)} \omega^{T}(s) \omega(s) d s \\
& \leq \cdots \leq \mu^{N} e^{a T^{-}(0, t)+\beta T^{+}(0, t)} V_{\sigma\left(t_{0}\right)}(0) \\
& \quad+\int_{0}^{t} e^{\pi_{\sigma(t)}(t-s)} \mu^{N} \kappa_{\sigma(t)} \omega^{T}(s) \omega(s) d s \\
& \leq \mu^{N}\left(e^{a\left(T-T^{+}(0, t)\right)+\beta T^{+}(0, t)} V_{\sigma\left(t_{0}\right)}(0)+\kappa e^{\beta T} d_{w}\right),
\end{aligned}
$$

where $a=\max _{i \in \mathcal{S}}\left(a_{i}\right), \beta=\max _{i \in \mathcal{S}}\left(\beta_{i}\right)$, and $\kappa=\max _{i \in \mathcal{S}}\left(\kappa_{i}\right)$. Consequently, it can be obtained that

$$
\begin{aligned}
\mathcal{E}^{T}(t) R \varepsilon(t) \leq & \lambda_{\max }(R) \varepsilon^{T}(t) \varepsilon(t) \leq \lambda_{\max }(R) \frac{V_{\sigma(t)}(t)}{\lambda_{\min }\left(P_{\sigma(t)}\right)} \\
< & \frac{\lambda_{\max }(R)}{\lambda_{\min }\left(P_{\sigma(t)}\right)} \mu^{N}\left(e^{a\left(T-T^{+}(0, t)\right)+\beta T^{+}(0, t)} V_{\sigma(t 0)}(0)\right. \\
& \left.+\kappa e^{\beta T} d w\right) \leq \frac{\lambda_{\max }(R) \lambda_{\max }\left(P_{\sigma(t)}\right)}{\lambda_{\min }\left(P_{\sigma(t)}\right)} \\
& \cdot \mu^{N}\left(e^{a\left(T-T^{+}(0, t)\right)+\beta T^{+}(0, t)} \varepsilon^{T}(0) \varepsilon(0)+\kappa e^{\beta T} d w\right) \\
\leq & \frac{\lambda_{\max }(R) \lambda_{\max }\left(P_{\sigma(t)}\right)}{\lambda_{\min }\left(P_{\sigma(t)}\right) \lambda_{\min }(R)} \mu^{N}\left(e^{a\left(T-T^{+}(0, t)\right)+\beta T^{+}(0, t)} c_{1}\right. \\
& \left.+\lambda_{\min }(R) \kappa e^{\beta T} d_{w}\right)<c_{2},
\end{aligned}
$$

which means that

$$
T^{+}(0, t)<\frac{\ln \left(c_{2} \lambda_{\min }\left(P_{i}\right) \lambda_{\min }(R)-\mu^{N} \lambda_{\max }(R) \lambda_{\max }\left(P_{i}\right) \lambda_{\min }(R) \kappa e^{\beta T} d_{w}\right)}{\beta-a}-\frac{N \ln \mu-\ln c_{1} \lambda_{\max }(R) \lambda_{\max }\left(P_{i}\right)-a T}{\beta-a}
$$

In addition, the ADT $\tau_{a}$ can be obtained for a fixed $T^{+}$ $(0, t)$ according to Definition 1 , which completes the proof.

Theorem 4. Consider the group of UAVs (1) with switching topologies and disturbances, for giving constants $\alpha_{i} \geq 0, \beta_{i} \geq$ $0, i \in \mathcal{S}, \mu>1, \kappa_{1 i}>0$, and $\kappa_{2 i}>0$, if there exist matrices $\tilde{P}_{i}>0$ and $W_{2 i}, \forall(i, j) \in \mathcal{S} \times \mathcal{S}, i \neq j$, such that

$$
\begin{gathered}
{\left[\begin{array}{cc}
\Phi_{1 i} & (I \otimes B) \\
* & -\kappa_{1 i} I
\end{array}\right]<0, \quad i \in \mathscr{A} \mathscr{T},} \\
{\left[\begin{array}{cc}
\Phi_{2 i} & (I \otimes B) \\
* & -\kappa_{2 i} I
\end{array}\right]<0, \quad i \in \mathscr{U} \mathscr{A} \mathscr{T},} \\
\tilde{P}_{i} \preceq \mu_{i} \tilde{P}_{j},
\end{gathered}
$$


Input: $A, B, L_{\sigma(t)}$

Output: $K_{2 \sigma(t)}$

1: Given constants $\alpha_{\sigma(t)}>0, \beta_{\sigma(t)}>0, \mu>1, \sigma(t) \in \mathcal{S}, \kappa_{1 i}>0, \kappa_{2 i}>0$;

2: Configure the formation centre by $A+B K_{1 \sigma(t)}$ with desired eigenvalues;

3: Obtaining $M_{\sigma(t)}$ by $L \sigma(t)=M \sigma(t) E$;

4: Solving the LMIs in Theorem 4;

5: Obtaining feasible solutions of $\tilde{P}_{\sigma(t)}>0$ and $W_{2 \sigma(t)}$;

6: Obtaining the total dwell time of the unachievable topologies $T^{+}$;

7: return $K_{2 \sigma(t)}=W_{2 \sigma(t)} \tilde{P}_{\sigma(t)}^{-1}$.

Algorithm 1: FTBF algorithm.

where

$$
\begin{aligned}
\Phi_{1 i}:= & \operatorname{sym}\left\{\left(I \otimes A \tilde{P}_{i}\right)+\left(I \otimes B K_{1 i} \tilde{P}_{i}\right)+\left(E M_{i} \otimes B W_{2 i}\right)\right\} \\
& -\alpha_{i}\left(I \otimes A \tilde{P}_{i}\right),
\end{aligned}
$$

$$
\begin{aligned}
\Phi_{2 i}:= & \operatorname{sym}\left\{\left(I \otimes A \tilde{P}_{i}\right)+\left(I \otimes B K_{1 i} \tilde{P}_{i}\right)+\left(E M_{i} \otimes B W_{2 i}\right)\right\} \\
& -\beta_{i}\left(I \otimes A \tilde{P}_{i}\right),
\end{aligned}
$$

and the total dwell time of the unachievable topologies $T^{+}$ satisfies

$$
T^{+}(0, t)<\frac{\ln \left(c_{2} \lambda_{\min }\left(\tilde{P}_{i}^{-1}\right) \lambda_{\min }(R)-\mu^{N} \lambda_{\max }(R) \lambda_{\max }\left(\tilde{P}_{i}^{-1}\right) \lambda_{\min }(R) \kappa e^{\beta T} d_{w}\right)}{\beta-a}-\frac{N \ln \mu-\ln c_{1} \lambda_{\max }(R) \lambda_{\max }\left(\tilde{P}_{i}^{-1}\right)-a T}{\beta-a}
$$

Then, the FTBF can be achieved with respect to $\left(c_{1}, c_{2}\right.$, $\left.T, R, \sigma(t), d_{w}\right)$.
Furthermore, when $T^{+}$can be fixed, the switching signal has an ADT satisfying

$$
\tau_{a}>\tau_{a}^{*}=\frac{T \ln \mu}{\ln c_{2} \lambda_{\min }\left(\tilde{P}_{i}^{-1}\right) \lambda_{\min }(R)-\ln \left(\lambda_{\max }(R) \lambda_{\max }\left(\tilde{P}_{i}^{-1}\right)\left(e^{a\left(T-T^{+}(0, t)\right)+\beta T^{+}(0, t)} c_{1}+\lambda_{\min }(R) \kappa e^{\beta T} d_{w}\right)\right)} .
$$

In addition, when the topology-dependent controller gain $K_{1 i}$ is configured, $K_{2 i}$ can be obtained by

$$
K_{2 i}=W_{2 i} \tilde{P}_{i}^{-1}
$$

Proof 4. By applying the same matrix congruent transformation in Theorem 2, the results can be directly obtained. follows.

Based on the above results, Algorithm 1 can be given as

Corollary 2. Consider the group of UAVs (1) with switching topologies and disturbances, for giving constants $\alpha_{i} \geq 0$ and $\beta_{i} \geq 0, i \in \mathcal{S}$, if there exist matrices $\tilde{P}_{i}>0$ and $W_{2 i}, \forall(i, j) \in$ $\mathcal{S} \times \mathcal{S}, i \neq j$, such that

$$
\begin{aligned}
& {\left[\begin{array}{cc}
\Phi_{1 i} & (I \otimes B) \\
* & -\kappa_{1 i} I
\end{array}\right]<0, \quad i \in \mathscr{A} \mathscr{T},} \\
& {\left[\begin{array}{cc}
\Phi_{2 i} & (I \otimes B) \\
* & -\kappa_{2 i} I
\end{array}\right]<0, \quad i \in \mathscr{U} \mathscr{A} \mathscr{T},}
\end{aligned}
$$

and the total dwell time of the unachievable topologies $T^{+}$ satisfies

$$
T^{+}(0, t)<\frac{\ln \left(c_{2} \lambda_{\min }\left(\tilde{P}_{i}^{-1}\right) \lambda_{\min }(R)-\lambda_{\max }(R) \lambda_{\max }\left(\tilde{P}_{i}^{-1}\right) \lambda_{\min }(R) \kappa e^{\beta T} d_{w}\right)}{\beta-a}-\frac{\ln c_{1} \lambda_{\max }\left(\tilde{P}_{i}^{-1}\right)-a T}{\beta-a} .
$$




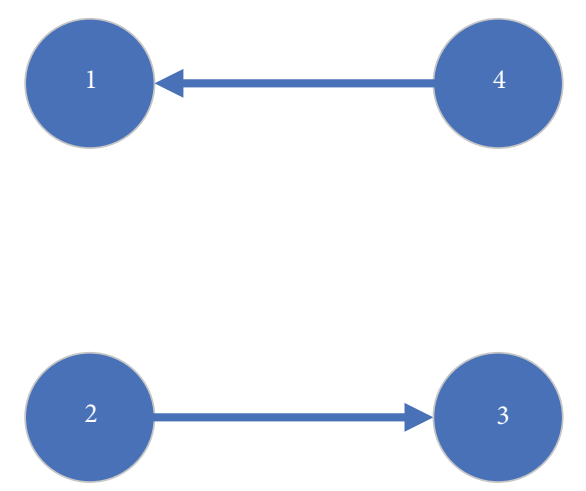

(a) Topology 1

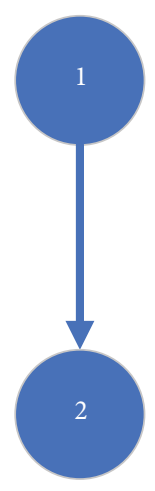

(b) Topology 2
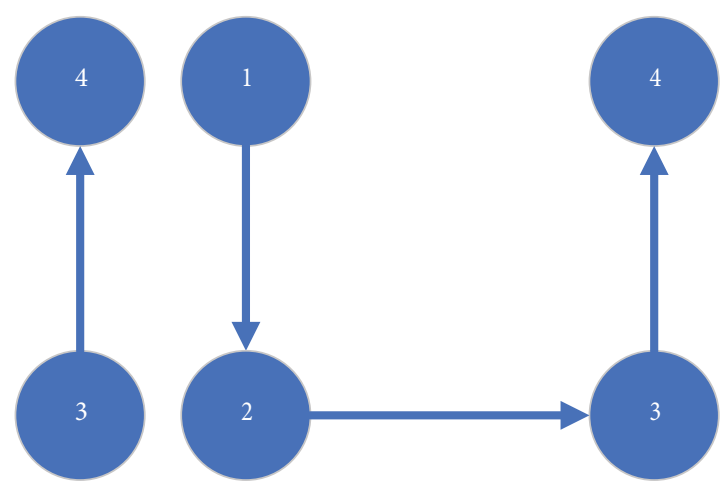

(c) Topology 3

FIgURE 1: The switching communication topologies of UAVs.

Then, the UFTBF can be achieved with respect to $\left(c_{1}, c_{2}\right.$, $\left.T, R, d_{w}\right)$.

In addition, when the topology-dependent controller gain $K_{1 i}$ is configured, $K_{2 i}$ can be obtained by

$$
K_{2 i}=W_{2 i} \tilde{P}_{i}^{-1}
$$

\section{Illustrative Example}

In the following, a numerical example is given to verify the effectiveness of our proposed formation design.

Consider a group of four UAVs with the following switching communication topologies depicted in Figure 1.

The corresponding Laplacian matrices can be given as follows:

$$
\begin{aligned}
L_{1} & =\left[\begin{array}{cccc}
1 & 0 & 0 & -1 \\
0 & 0 & 0 & 0 \\
0 & -1 & 1 & 0 \\
0 & 0 & 0 & 0
\end{array}\right], \\
L_{2} & =\left[\begin{array}{cccc}
0 & 0 & 0 & 0 \\
-1 & 1 & 0 & 0 \\
0 & 0 & 0 & 0 \\
0 & 0 & -1 & 1
\end{array}\right], \\
L_{3} & =\left[\begin{array}{cccc}
0 & 0 & 0 & 0 \\
-1 & 1 & 0 & 0 \\
0 & -1 & 1 & 0 \\
0 & 0 & -1 & 1
\end{array}\right] .
\end{aligned}
$$

It can be verified that the formation cannot be achieved since topology 1 and topology 2 do not have directed spanning trees, which implies that they are unachievable.
Accordingly, it can be obtained that

$$
M_{1}=\left[\begin{array}{ccc}
1 & 1 & 1 \\
0 & 0 & 0 \\
0 & -1 & 0 \\
0 & 0 & 0
\end{array}\right],
$$

$$
M_{2}=\left[\begin{array}{ccc}
0 & 0 & 0 \\
-1 & 0 & 0 \\
0 & 0 & 0 \\
0 & 0 & -1
\end{array}\right] \text {, }
$$

$$
M_{3}=\left[\begin{array}{ccc}
0 & 0 & 0 \\
-1 & 0 & 0 \\
0 & -1 & 0 \\
0 & 0 & -1
\end{array}\right] \text {. }
$$

The time-varying formation configuration is given as $h_{i}(t)=\left[3 \sin t, 0.6 \cos t, \mathrm{e}_{i} \cos t, 3 \cos t,-0.6 \sin t,-\sin t\right]^{T}$, $\mathrm{Q}_{1}=1, \mathrm{\varrho}_{2}=0.8, \mathrm{\varrho}_{3}=0.6$, and $\mathrm{\varrho}_{4}=0.4$, and the external disturbances are set by $0.005 \sin t$.

The design parameters are chosen as $\alpha=0.1, \beta=0.5$, $\mu=1.3, \kappa_{13}=0.06, \kappa_{21}=0.02$, and $\kappa_{22}=0.03$. For the FTBF, the parameter $c_{1}$ is chosen as 0.5 , the parameter $c_{2}$ is set as $50, T$ is given by $10 \mathrm{~s}$, and $d_{w}$ is supposed to be 0.0025 .

The eigenvalues of $I \otimes(A+B K)$ are configured by $(-1 \pm i$, $-1 \pm i$, and $-1 \pm i)$, one has

$$
K_{1 i}=\left[\begin{array}{cccccc}
-2 & 0 & 0 & -2 & 0 & 0 \\
0 & -2 & 0 & 0 & -2 & 0 \\
0 & 0 & -2 & 0 & 0 & -2
\end{array}\right] .
$$

Based on the established results of Theorem 4, it can be obtained by solving the LMIs that 


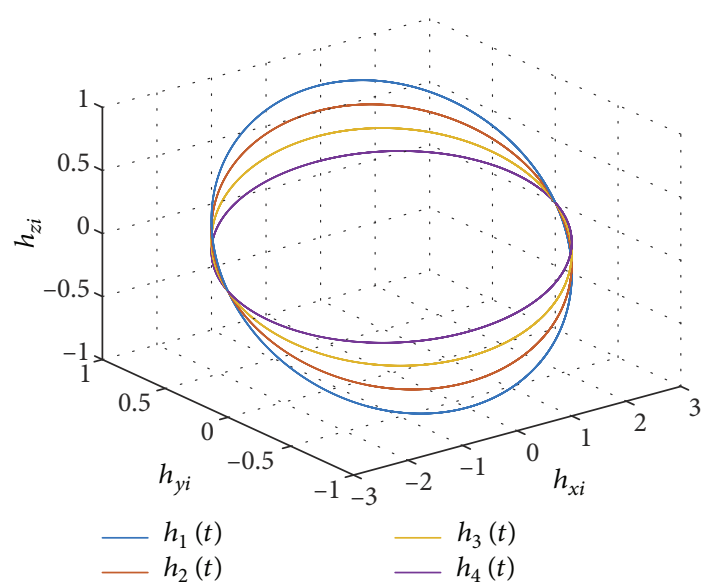

FIGURE 2: State trajectories of desired time-varying formation.

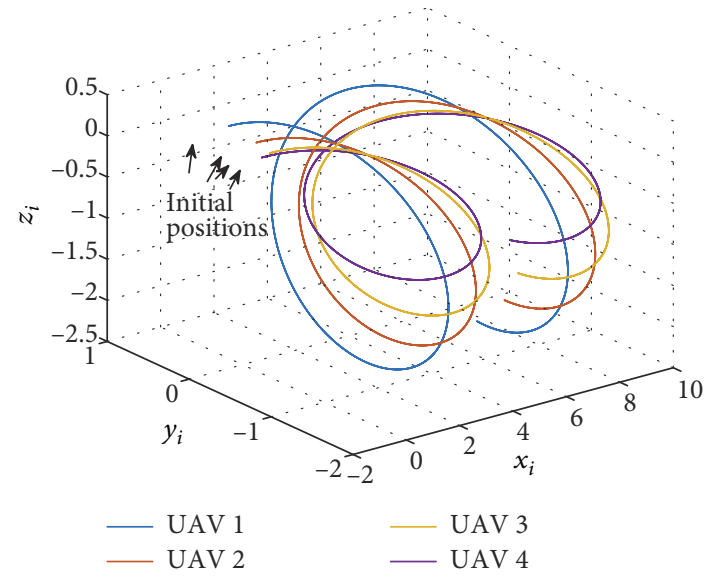

FIGURE 3: State trajectories of the open-loop FTBF trajectory.

$$
\begin{aligned}
K_{21} & =\left[\begin{array}{cccccc}
0.3424 & 0 & 0 & 0.5493 & 0 & 0 \\
0 & 0.3424 & 0 & 0 & 0.5493 & 0 \\
0 & 0 & 0.3424 & 0 & 0 & 0.5493
\end{array}\right], \\
K_{22} & =\left[\begin{array}{cccccc}
0.4996 & 0 & 0 & 0.7677 & 0 & 0 \\
0 & 0.4996 & 0 & 0 & 0.7677 & 0 \\
0 & 0 & 0.4996 & 0 & 0 & 0.7677
\end{array}\right], \\
K_{23} & =\left[\begin{array}{cccccc}
0.4310 & 0 & 0 & 0.5133 & 0 & 0 \\
0 & 0.4310 & 0 & 0 & 0.5133 & 0 \\
0 & 0 & 0.4310 & 0 & 0 & 0.5133
\end{array}\right],
\end{aligned}
$$

and the total dwell time of the unachievable topologies $T^{+}$is calculated as $4.04 \mathrm{~s}$.

In the simulation, the initial conditions of the UAVs are given as $[0.1 ; 0.2 ; 0.3 ; 0 ; 0 ; 0 ;-0.1 ;-0.2 ; 0.3 ; 0 ; 0 ; 0 ; 0.1$; $-0.3 ; 0.2 ; 0 ; 0 ; 0 ; 0.1 ;-0.2 ; 0.1 ; 0 ; 0 ; 0]^{T}$ and $T^{+}$is set as $2.5 \mathrm{~s}$. Figures $2-4$ show the desired time-varying formation trajectory, the open-loop FTBF trajectory, and the closedloop FTBF trajectory, respectively. It can be seen that the designed formation controllers for each UAV can achieve

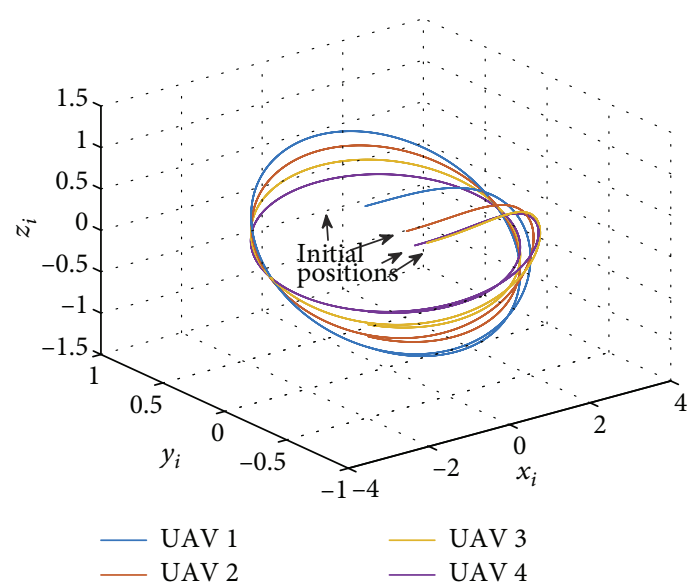

FIgURE 4: State trajectories of the closed-loop FTBF trajectory.

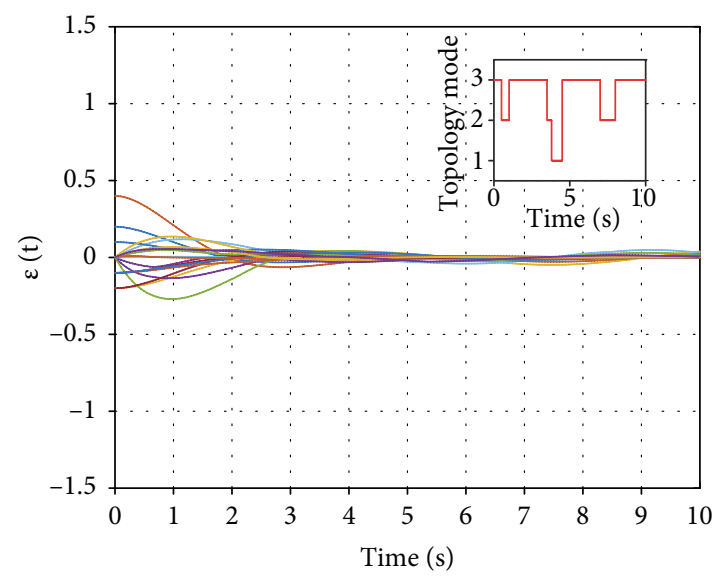

FIgURE 5: State trajectories of formation errors.

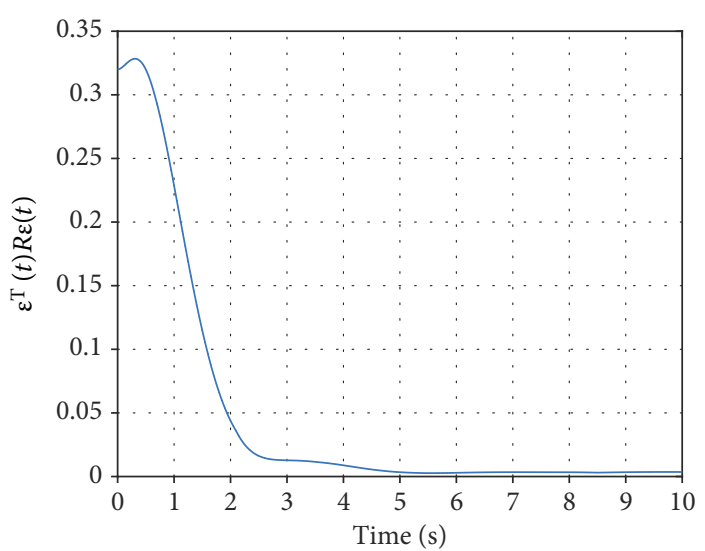

Figure 6: State trajectories of $\varepsilon^{T}(t) R \varepsilon(t)$.

the prescribed formation with unachievable topologies and external disturbances. Figure 5 depicts the formation errors of the UAVs with the finite-time interval, and Figure 6 indicates the corresponding FTBF conditions. It can be found that the formation errors are finite-time bounded with switching topologies according to Definition 4 with $\varepsilon^{T}(0) R \varepsilon$ $(0)=0.32$ and $\varepsilon^{T}(10) R \varepsilon(10)=0.002$, which supports our theoretical analysis. 


\section{Conclusion}

In this paper, the formation problem of UAVs with switching topologies and disturbance is concerned with an ADT approach. In particular, the concept of FTBF is introduced to deal with the finite-time stability problem and disturbances. Moreover, both achievable and unachievable topologies are taken into account for a more practical background of communications among the UAVs. Based on model transformation, sufficient conditions are established for the FTBF and the desired topology-dependent controller can be designed accordingly. In the end, a numerical example is given to demonstrate the effectiveness of our proposed control method. Our future study will focus on the cases with model uncertainties and communication delays.

\section{Data Availability}

All data used to support the findings of this study are included within the article.

\section{Conflicts of Interest}

The authors declare that they have no conflicts of interest.

\section{Acknowledgments}

This work was supported by the National Natural Science Foundation of China under Grant 61703038, 61627808, and 9164820, the Fundamental Research Funds for the Central Universities under Grant FRF-TP-18-034A2, and the National Key Research and Development Program of China under Grant SQ2017YFB130092. This work is also supported by the Strategic Priority Research Program of the CAS (Grant XDB02080003).

\section{References}

[1] K. P. Valavanis, Advances in Unmanned Aerial Vehicles: State of the Art and the Road to Autonomy, Vol. 33, Springer Science \& Business Media, 2008.

[2] D. M. Stipanovic, G. Inalhan, R. Teo, and C. J. Tomlin, "Decentralized overlapping control of a formation of unmanned aerial vehicles," Automatica, vol. 40, no. 8 , pp. 1285-1296, 2004.

[3] X. Dong, B. Yu, Z. Shi, and Y. Zhong, "Time-varying formation control for unmanned aerial vehicles: theories and applications," IEEE Transactions on Control Systems Technology, vol. 23, no. 1, pp. 340-348, 2015.

[4] Z. Peng, G. Wen, S. Yang, and A. Rahmani, "Distributed consensus-based formation control for nonholonomic wheeled mobile robots using adaptive neural network," Nonlinear Dynamics, vol. 86, no. 1, pp. 605-622, 2016.

[5] S. Yang, Y. Cao, Z. Peng, G. Wen, and K. Guo, "Distributed formation control of nonholonomic autonomous vehicle via RBF neural network," Mechanical Systems and Signal Processing, vol. 87, pp. 81-95, 2017.

[6] A. Abdessameud and A. Tayebi, "Formation control of VTOL unmanned aerial vehicles with communication delays," Automatica, vol. 47, no. 11, pp. 2383-2394, 2011.
[7] J. Wang and M. Xin, "Integrated optimal formation control of multiple unmanned aerial vehicles," IEEE Transactions on Control Systems Technology, vol. 21, no. 5, pp. 1731-1744, 2013.

[8] Z. Chao, S.-L. Zhou, L. Ming, and W.-G. Zhang, "UAV formation flight based on non-linear model predictive control," Mathematical Problems in Engineering, vol. 2012, Article ID 261367, 15 pages, 2012.

[9] Y. Kuriki and T. Namerikawa, "Formation control with collision avoidance for a multi-UAV system using decentralized MPC and consensus-based control," SICE Journal of Control, Measurement, and System Integration, vol. 8, no. 4, pp. 285294, 2015.

[10] A. Ryan, M. Zennaro, A. Howell, R. Sengupta, and J. K. Hedrick, "An overview of e205 merging results in cooperative UAV control," in 2004 43rd IEEE Conference on Decision and Control (CDC) (IEEE Cat. No.04CH37601), pp. 602-607, Nassau, Bahamas, December 2004.

[11] H. Chao, Y. Cao, and Y. Chen, "Autopilots for small unmanned aerial vehicles: a survey," International Journal of Control, Automation and Systems, vol. 8, no. 1, pp. 36-44, 2010.

[12] S. Hayat, E. Yanmaz, and R. Muzaffar, "Survey on unmanned aerial vehicle networks for civil applications: a communications viewpoint," IEEE Communications Surveys \& Tutorials, vol. 18, no. 4, pp. 2624-2661, 2016.

[13] M. Mozaffari, W. Saad, M. Bennis, and M. Debbah, "Unmanned aerial vehicle with underlaid device-to-device communications: performance and tradeoffs," IEEE Transactions on Wireless Communications, vol. 15, no. 6, pp. 3949 3963, 2016.

[14] M. Tortonesi, C. Stefanelli, E. Benvegnu, K. Ford, N. Suri, and M. Linderman, "Multiple-UAV coordination and communications in tactical edge networks," IEEE Communications Magazine, vol. 50, no. 10, pp. 48-55, 2012.

[15] D. Dionne and C. A. Rabbath, "Multi-UAV decentralized task allocation with intermittent communications: the DTC algorithm," in 2007 American Control Conference, pp. 54065411, New York, NY, USA, July 2007.

[16] X. Dong, Y. Zhou, Z. Ren, and Y. Zhong, "Time-varying formation control for unmanned aerial vehicles with switching interaction topologies," Control Engineering Practice, vol. 46, pp. 26-36, 2016.

[17] M. Mesbahi and F. Y. Hadaegh, "Formation flying control of multiple spacecraft via graphs, matrix inequalities, and switching," Journal of Guidance, Control, and Dynamics, vol. 24, no. 2, pp. 369-377, 2001.

[18] Y. Qi, S. Zhou, Y. Kang, and S. Yan, "Formation control for unmanned aerial vehicles with directed and switching topologies," International Journal of Aerospace Engineering, vol. 2016, Article ID 7657452, 8 pages, 2016.

[19] X. Zhao, L. Zhang, P. Shi, and M. Liu, "Stability of switched positive linear systems with average dwell time switching," Automatica, vol. 48, no. 6, pp. 1132-1137, 2012.

[20] J. Zhao, D. J. Hill, and T. Liu, "Synchronization of complex dynamical networks with switching topology: a switched system point of view," Automatica, vol. 45, no. 11, pp. 25022511, 2009.

[21] F. J. Zhong and Y. C. Zhao, "Stability analysis switched systems," Applied Mechanics and Materials, vol. 389, pp. 685691, 2013. 
[22] H. Lin and P. J. Antsaklis, "Stability and stabilizability of switched linear systems: a survey of recent results," IEEE Transactions on Automatic Control, vol. 54, no. 2, pp. 308322, 2009.

[23] X. Zhao, S. Yin, H. Li, and B. Niu, "Switching stabilization for a class of slowly switched systems," IEEE Transactions on Automatic Control, vol. 60, no. 1, pp. 221-226, 2015.

[24] X. Zhao, L. Zhang, P. Shi, and M. Liu, "Stability and stabilization of switched linear systems with mode-dependent average dwell time," IEEE Transactions on Automatic Control, vol. 57, no. 7, pp. 1809-1815, 2012.

[25] G. Zhai, B. Hu, K. Yasuda, and A. N. Michel, "Stability analysis of switched systems with stable and unstable subsystems: an average dwell time approach," International Journal of Systems Science, vol. 32, no. 8, pp. 1055-1061, 2001.

[26] X. Chen, K. Zhang, and B. Jiang, "Robust finite-time fault diagnosis for leader-follower multi-agent systems," in 2017 29th Chinese Control And Decision Conference (CCDC), pp. 303308, Chongqing, China, May 2017.

[27] X. Liu, J. Lam, W. Yu, and G. Chen, "Finite-time consensus of multiagent systems with a switching protocol," IEEE Transactions on Neural Networks and Learning Systems, vol. 27, no. 4, pp. 853-862, 2016.

[28] R. Sakthivel, S. Kanakalakshmi, B. Kaviarasan, Y.-K. Ma, and A. Leelamani, "Finite-time consensus of input delayed multiagent systems via non-fragile controller subject to switching topology," Neurocomputing, vol. 325, pp. 225-233, 2019.

[29] W. Yu, G. Chen, and M. Cao, "Some necessary and sufficient conditions for second-order consensus in multi-agent dynamical systems," Automatica, vol. 46, no. 6, pp. 1089-1095, 2010.

[30] C. Ma and W. Wu, "Distributed leader-follower consensus of nonlinear multiagent systems with unconsensusable switching topologies and its application to flexible-joint manipulators," Systems Science \& Control Engineering, vol. 6, no. 3, pp. 200207, 2018.

[31] J. P. Hespanha and A. S. Morse, "Stability of switched systems with average dwell-time," in Proceedings of the 38th IEEE Conference on Decision and Control (Cat. No.99CH36304), pp. 2655-2660, Phoenix, AZ, USA, December 1999.

[32] H. Liu, Y. Shen, and X. Zhao, "Delay-dependent observerbased $h \infty$ finite-time control for switched systems with timevarying delay," Nonlinear Analysis: Hybrid Systems, vol. 6, no. 3, pp. $885-898,2012$. 


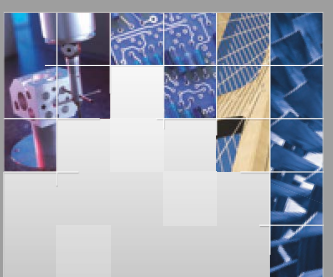

\section{Enfincering}
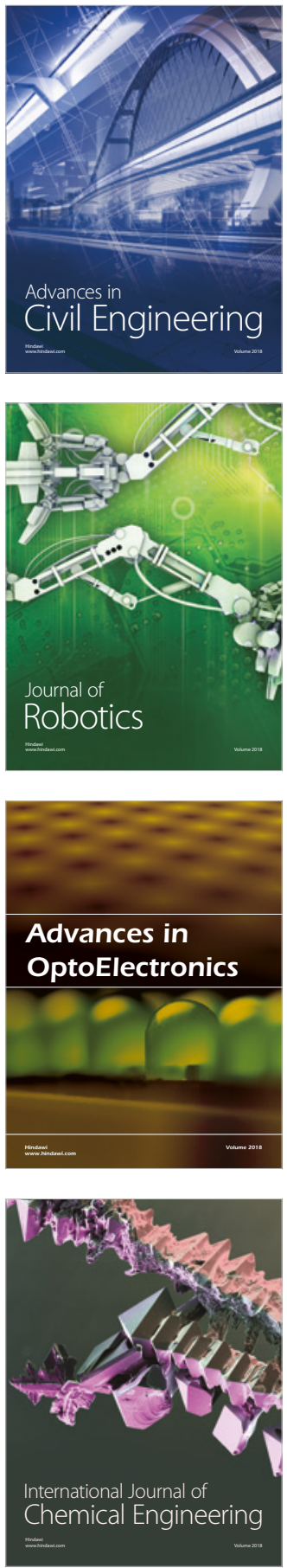

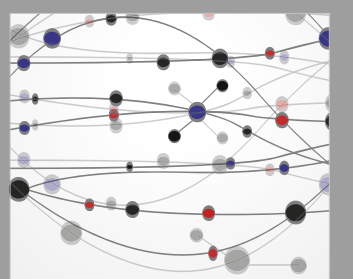

\section{Rotating \\ Machinery}

The Scientific World Journal

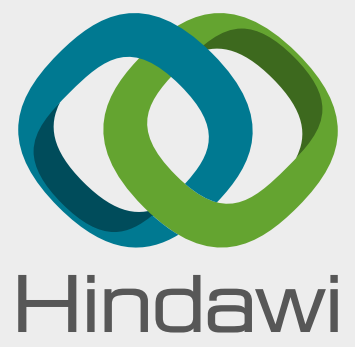

Submit your manuscripts at

www.hindawi.com
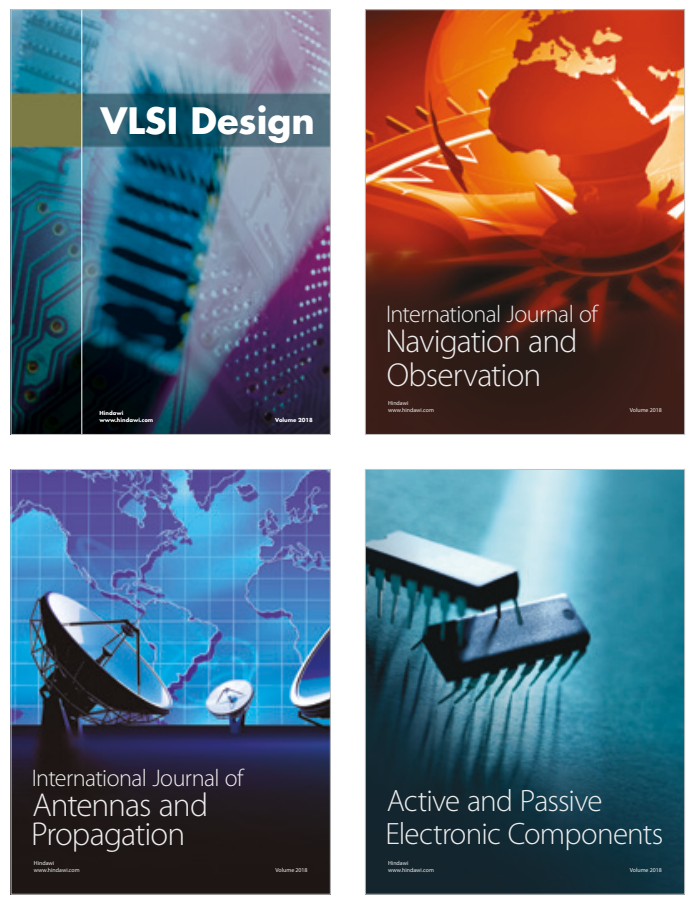
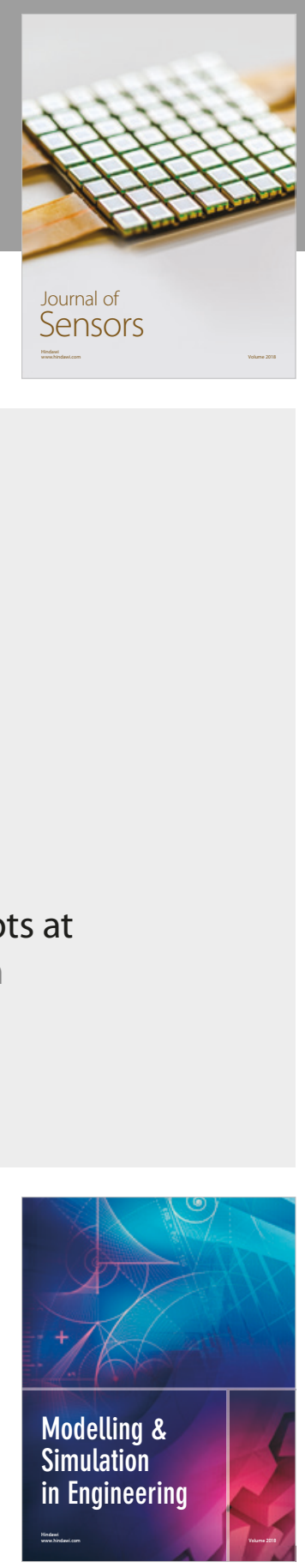

\section{Advances \\ Multimedia}
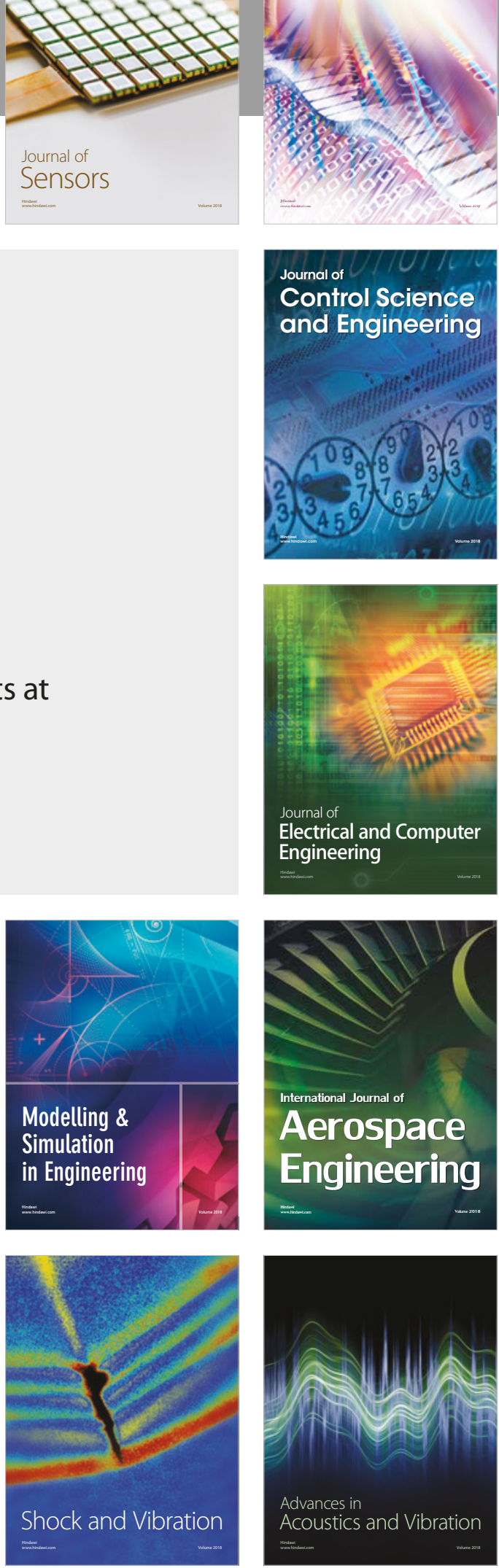